\title{
Lectin-Binding Domains on Laminin
}

\author{
C. N. RAO,* I. J. GOLDSTEIN,† AND L. A. LIOTTA*,I
}

\begin{abstract}
*Laboratory of Pathology, National Cancer Institute, National Institutes of Health, Bethesda, Maryland 20205, and $\dagger$ Department of Biological Chemistry, University of Michigan, Ann Arbor, Michigan 48109
\end{abstract}

Received March 28, 1983, and in revised form July 11, 1983

The nature and location of carbohydrate moieties on the laminin molecule were identified by studying the binding affinity of a series of lectins for purified, proteasederived fragments of laminin. Laminin is a cross-shaped molecule containing three short arms $(36 \mathrm{~nm})$ and one long arm $(76 \mathrm{~nm})$. All arms contain globular end regions by electron microscopy. Purified fragments of laminin were obtained which (a) lacked the long arm of the molecule but retained the intact short arms, or (b) lacked both the long arm and the globular end regions of the short arms. These two types of fragments differed markedly in lectin-binding capacity. Using the known sugar specificities of the lectins and hapten sugar competition for lectin-binding to laminin fragments, the following conclusions were reached: (a) $\alpha$-D-Galactopyranosyl end groups are markedly enriched in the globular end regions of the short arms compared to the rod-shaped portions of the molecule. (b) $\alpha$-D-Mannopyranosyl residues are present on both the globular end regions and the rod-shaped portions of the molecule. (c) Exposed $\mathrm{N}$-acetyl-D-galactosaminyl end groups are absent or present in low amounts on laminin. (d) (NANA)- $(2 \rightarrow 6)-\beta$-D-Gal- $(1 \rightarrow 4)-\beta$-D-GlcNAc-(1 $\rightarrow 2)-\mathrm{D}-\mathrm{Man}$-terminated oligosaccharide units are enriched on the rod-shaped regions of the short arms compared to the globular end regions.

Laminin, a glycoprotein of basement membranes, mediates cell attachment and also binds to other types of matrix components. Laminin mediates the attachment of both normal and neoplastic cells of mesenchymal and epithelial origin (1). Laminin also has been found to bind to proteoglycan and type IV collagen (2-4). A cell plasma membrane receptor for laminin has been identified and isolated $(5,6)$.

Laminin is a large molecule $\left(M_{\mathrm{r}}\right.$ $=1,000,000)$ with an unusual cross-shaped structure $(7,8)$. By electron microscopy laminin contains three short arms $(36 \mathrm{~nm})$ and one long arm $(76 \mathrm{~nm})$. All arms contain globular end regions $(7,8)$. It has been hy-

\footnotetext{
${ }^{1}$ To whom correspondence should be addressed: Chief, Laboratory of Pathology, Building 10, Room 2A33, National Cancer Institute, National Institutes of Health, Bethesda, Md. 20205.
}

pothesized that the four-arm structure of laminin is of biological importance enabling the molecule to "reach out" and interact with multiple cellular and matrix components in different directions (9). Such a diversity of binding regions on the laminin molecule would be expected to reflect biochemical differences among the molecular domains. Although it was previously shown that murine laminin binds to Griffomia simplicifolia $\mathrm{I}-\mathrm{B}_{4}$ isolectin (10) the location of the lectin-binding region on the laminin molecule was not identified. We therefore undertook to study whether domains of the laminin molecule exhibited differences in exposed lectin-binding carbohydrate residues. In particular, we aimed to compare the carbohydrate composition of the globular end regions with that of the rod-shaped portion of the short arms of the laminin molecule. 
Protease-derived fragments of laminin were prepared which (a) lacked the long arm but retained the intact short arms, or (b) lacked both the long arm and the globular end regions of the short arms. The binding affinity of these fragments to lectins immobilized on a solid phase was then studied for a variety of lectins with known sugar-binding specificities. The specificity of the binding was verified by using defined haptenic sugars to compete for binding. The results of these studies indicated a marked qualitative difference in the lectinbinding capacity of the laminin fragments.

\section{MATERIALS AND METHODS}

Purification of laminin and laminin fragments. Laminin was purified from $0.5 \mathrm{M} \mathrm{NaCl}$ extracts of mouse EHS $^{2}$ tumor by DEAE-cellulose and agarose A 5M column chromatography and stored frozen in phosphate-buffered saline (11).

Protease-derived fragments of laminin were purified and examined by electron microscopy as described previously $(8,12,13)$. The domains of the molecule retained in the fragments are summarized in Fig. 7. The $\alpha$ fragment generated by digestion with $\alpha$ thrombin lacks the long arm but retains the three short arms with globular end regions. The $C_{1}$ and $P_{1}$ fragments generated by chymotrypsin and pepsin, respectively, lack both the long arm and the globular end regions of the short arms. The $\mathrm{C}_{1}$ fragment shown in Fig. 7 has a "T"' structure with arm lengths of 32 $n m$. The $P_{1}$ fragment is similar in appearance but has shorter arm lengths $(26 \mathrm{~nm})(7,8,12)$. Homogeneity of laminin and the three fragments was verified by electrophoresis on $5 \%$ slab gels according to Laemmli (14) in the presence of $0.5 \mathrm{M}$ urea.

Iodination of laminin and laminin fragments. Laminin and the three fragments were iodinated by the lacto-peroxidase method (15). The reagents and the unreacted iodine were removed from the iodinated proteins by molecular-sieve chromatography.

Binding assay. The GS I-B ${ }_{4}$ isolectin and the GS II lectin were isolated from $G$. simplicifolia seeds by the

\footnotetext{
${ }^{2}$ Abbreviations used: NC, nitrocellulose; PBS, phosphate-buffered saline, $+\mathrm{Ca}^{2+}$ and $\mathrm{Mg}^{2+}$; Con $\mathrm{A}$, concanavalin A; WGA, wheat germ agglutinin, GS I$\mathrm{B}_{4}$ : G. simplicifolia $\mathrm{I}-\mathrm{B}_{4} ; \mathrm{PHA}, P$. vulgaris agglutinin; GS II, G. simplicifolia II; LBL I, LBL II, and LBL III, Lima bean lectins I, II, and III; EHS, Engelbreth-Holm-Swarm; BSA, bovine serum albumin; GalNAc, $N$-acetylgalactosamine; GlcNAc, $N$-acetylglucosamine.
}

method of Delmotte and Goldstein (16). PHA lectins, concanavalin $\mathrm{A}$, wheat germ agglutinin, $N$-acetyl-Dgalactosamine, and $N$-acetyl-D-glucosamine were purchased from Sigma Chemical Company. Lima bean lectins I, II, and III were isolated by the procedure of Roberts and Goldstein (17). The Dolichos biflom lectin was the gift of Dr. Marilynn Etzler, University of California, Davis. The Datura stramonium lectin was prepared by the procedure of Crowley and Goldstein (18). Methyl- $\alpha$-D-galactopyranoside, methyl- $\beta$ D-galactopyrannoside, methl- $\alpha$-D-mannoside, methyl$\alpha-\mathrm{D}$-glucopyranoside, and methyl- $\beta$-D-glucopyranoside were gifts from Dr. Gilhert Ashwell (NIADDK, NIH). Nitrocellulose SCWP 8- $\mu \mathrm{m}$ filters and other types of filters were purchased from the Millipore Corporation (Bedford, Mass.).

The binding of laminin and laminin fragments to lectins immobilized on nitrocellulose was carried out as follows. The lectin to be studied for binding properties was dissolved in phosphate-buffered saline (+ calcium + magnesium), pH 7.4 (PBS). The lectinPBS solution was applied directly to the surface of nitrocellulose filters (13 mm dia. 8- $\mu \mathrm{m}$-pore-size Millipore SCWP type) resting on a hydrophobic plastic surface. A solution volume of $20 \mu \mathrm{l}$ applied by pipet was enough to completely soak the entire area of the filter without excess fluid accumulating. After incubation in a humidified chamber for $1 \mathrm{~h}$, the filters were immersed in a blocking solution of $3 \%$ bovine serum albumin (BSA/PBS: $2 \times 90 \mathrm{~min}$ ) and rinsed with PBS ( $2 \times 30 \mathrm{~min})$. The blocked filters were saturated with ${ }^{125}$ I-labeled whole laminin or the laminin fragments and incubation was conducted in a humidified chamber at $25^{\circ} \mathrm{C}$ for $30 \mathrm{~min}$. The filters were washed with PBS $(2 \times 45 \mathrm{~min})$ and placed in a Packard $\gamma$-counter to measure the bound radioactivity. The radioactivity bound to $\mathrm{NC}$ filters blocked with $3 \%$ BSA alone was subtracted as background (8-15\%) to give the final value for bound radioactivity. Carbohydrate specificity of the lectin-laminin binding was studied by two means: In the first method, the laminin-lectin-binding reaction was conducted in the presence of excess haptenic sugars, and in the second method the haptenic sugars were added at the end of the binding reaction and the radioactivity was eluted from the filters over $18 \mathrm{~h}$ at $25^{\circ} \mathrm{C}$.

\section{RESULTS}

The affinity of whole laminin for a series of lectins was studicd under conditions in which an excess of lectin was bound to nitrocellulose filters. When a series of lectin concentrations were bound to the $\mathrm{NC}$, the application of 10 or $20 \mu \mathrm{g}$ of lectin on the $\mathrm{NC}$ filter was in the plateau range of the laminin-binding curve (Fig. 1). Time-course 


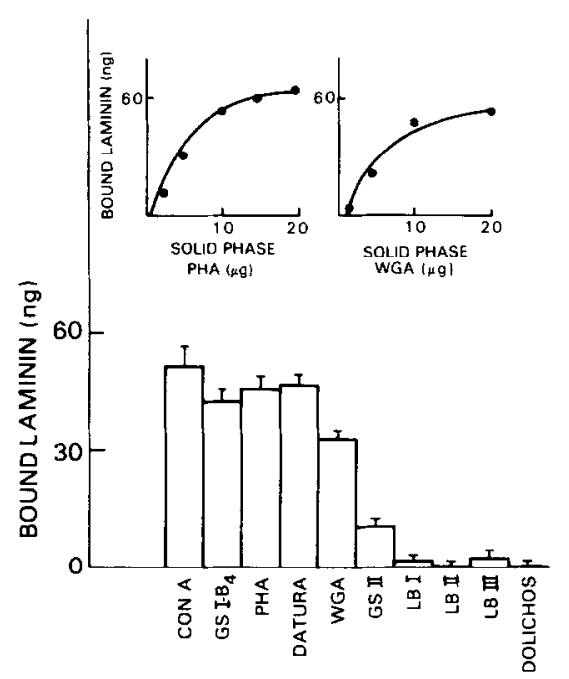

SOLID PHASE LECTIN

FIG. 1. Binding of ${ }^{125} \mathrm{I}$-laminin (specific activity 2500 $\mathrm{cpm} / \mathrm{ng})$ to Con A (10 $\mu \mathrm{g})$, GS I-B $(20 \mu \mathrm{g})$, PHA (20 $\mu \mathrm{g})$, Datura lectin $(20 \mu \mathrm{g})$, WGA $(20 \mu \mathrm{g})$, GS II (20 $\mu \mathrm{g})$, LB I, LB II, and LB III ( $8 \mu \mathrm{g})$, and Dolichos lectin $(20 \mu \mathrm{g})$. Inserts: The binding of laminin to PHA and WGA at different concentrations was plotted. Each point represents the mean of nine filters in three separate experiments and the values differed only by $10 \%$ between the filters. The nonspecific binding to BSA was $12 \%$.

studies showed that the rate of laminin binding reached a plateau after $20 \mathrm{~min}$. A variety of nitrocellulose filter types were compared for their lectin-binding properties. Using iodinated ligand, Millipore type SCWP bound nearly $100 \%$ of applied radioactivity and exhibited a background of laminin binding to BSA-blocked filters of $10 \%$. In contrast, NC filters type EAWP, HVLP, HVHP (Millipore), Schleicher and Schuell, or Bethesda Research Labs bound only 20 to $50 \%$ of the applied radioactivity, and showed a background of laminin binding to BSA-blocked filters in the range of 50 to $80 \%$. The SCWP type was therefore clearly superior and was used for all subsequent experiments.

Whole laminin was observed to bind significantly to five out of the ten lectins studied. For these five lectins (Con A, GS I-B ${ }_{4}$, PHA, Datura, or WGA), up to $60 \%$ of the applied laminin bound to a molar excess $(>10 \times)$ of the lectin. Reduced binding to
GS II lectin was found. The LBL components I, II, and III and the D. biflorus lectin failed to bind laminin compared to background. As shown in Fig. 1, the variability in the binding assays was low.

The sugar-binding specificity for those lectins which had an affinity for laminin was studied both by competition and elution (extraction) with specific haptenic sugars. Specificity results from both methods were identical (Figs. 2-4).

Methyl - $\alpha$-D - mannopyranoside and methyl $\alpha$-D-glucopyranoside specifically inhibited laminin binding to Con A whereas methyl- $\alpha$ - and $\beta$-D-galactopyranoside, and $N$-acetyl-D-galactosamine were ineffective. Laminin binding to GS $\mathrm{I}^{-B_{4}}$ lectin was blocked effectively by methyl$\alpha-D$-galactopyranoside but not by methyl$\beta$-D-galactopyranoside. Laminin binding to PHA lectin and wheat germ agglutinin was specifically inhibited by $N$-acetyl-D-galactosamine and $N$-acetyl-D-glucosamine, respectively. Extraction of the filters with $50 \mathrm{~mm}$ sugar solutions showed a similar specificity. In the extraction studies methyl $\alpha$-D-mannopyranoside and methyl$\alpha$-D-glucopyranoside extracted most of the bound activity from the Con A-treated filters. Correspondingly, extraction with methyl- $\alpha$-D-galactopyranoside eluted laminin from the GS $\mathrm{I}_{-} \mathrm{B}_{4}$-treated filters and

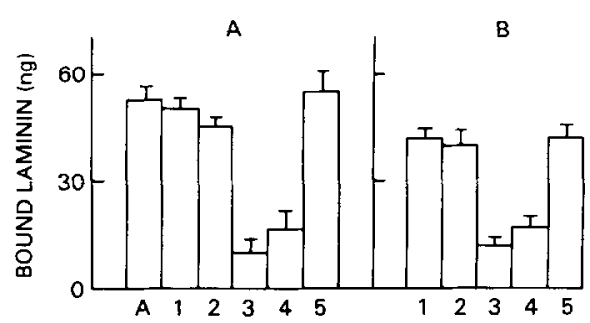

FIG. 2. (A) Influence of $50 \mathrm{~mm}$ solutions of (1) methyl- $\alpha$-D-galactopyranoside; (2) methyl- $\beta$-D-galactopyranoside; (3) methyl- $\alpha$-D-mannopyranoside; (4) methyl- $\alpha$-D-glucopyranoside, and $0.5 \mathrm{M}$ solution of (5) $\mathrm{N}$-acetyl-D-galactosamine on the binding of (A) ${ }^{125} \mathrm{I}$ laminin binding to $10 \mu \mathrm{g}$ of Con A. Haptens 3 and 4 significantly inhibited the binding while the other sugars were ineffective. (B) ${ }^{125}$ I-laminin bound to Con A was extracted with $50 \mathrm{~mm}$ solutions of haptens 14 and $0.5 \mathrm{M}$ solution of hapten 5 . Sugars 3 and 4 extracted 50 to $70 \%$ of the bound laminin. 


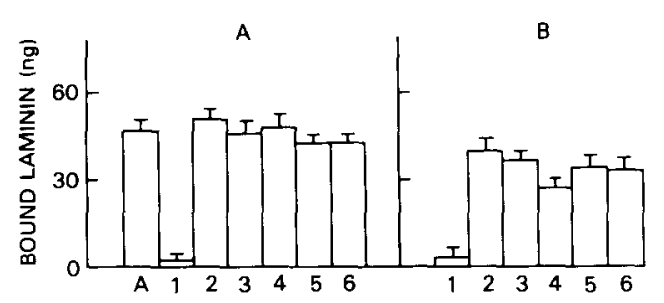

FIG. 3. (A) Influence of $50 \mathrm{~mm}$ solutions of haptens 1-4 and $0.5 \mathrm{M}$ solutions of haptens 5 and 6 on the binding of ${ }^{125} \mathrm{I}-$ laminin to $20 \mu \mathrm{g}$ of GS I-B $\mathrm{B}_{4}$ lectin (A). Methyl- $\alpha$-D-galactopyranoside (1) competed for the laminin binding to GS I-B 4 lectin. Haptens (2) methyl$\beta$-D-glucoside; (3) methyl- $\alpha$-D-mannoside; (4) methyl$\alpha$-D-glucoside; (5) $N$-acetyl-D-galactosamine, and (6) $N$-acetyl-D-glucosamine were ineffective. (B) Hapten methyl- $\alpha$-D-galactopyranoside (1) extracted significant amounts of bound laminin compared to sugars 2-6.

extraction with $N$-acetyl-D-galactosamine eluted laminin from the PHA lectin-coated filters. $N$-Acetyl-D-glucosamine specifically eluted the bound activity from wheat germ agglutinin-coated filters.

The laminin fragments differed in their lectin-binding properties (Figs. 5, 6). The $\alpha$-thrombin-derived fragment, missing the long arm of the molecule, bound to Con $A$, GS I-B ${ }_{4}$, PHA, and WGA. The $\alpha$ fragment exhibited somewhat reduced binding to

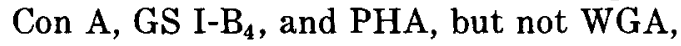
compared to whole laminin. The $C_{1}$ and $P_{1}$ fragments of laminin, which are missing the globular end regions, showed significantly reduced binding compared to whole laminin for Con $\mathrm{A}$. The $\mathrm{C}_{1}$ and $\mathrm{P}_{1}$ fragments failed to bind significantly to the GS I- $\mathrm{B}_{4}$ lectin. In contrast, the $C_{1}$ and $P_{1}$ fragments did not differ significantly from the $\alpha$ fragment in binding to WGA and PHA. These data indicate that the GS I- $\mathrm{B}_{4}$-binding domains, but not the PHA- or WGA-binding domains, are enriched on the globular ends of all four arms of the molecule.

\section{DISCUSSION}

In this report, a new lectin-binding assay is described to study the lectin-binding domains on laminin. The nitrocellulose-filter assay is simple, rapid, reproducible, and exhibits appropriate specificity. With a molar excess ratio of bound lectin to applicd laminin, whole laminin bound well to only five out of the ten lectins tested. This qualititative difference indicates that laminin is rich in some carbohydrate residues but lacks others. Of all the lectins tested, concanavalin A bound to the highest extent, indicating that laminin is probably rich in $\alpha$-D-mannopyranosyl residues.

Precipitin reactions between concanavalin A and glycoproteins are specifically inhibited by methyl- $\alpha$-D-mannopyranoside and to a lesser extent by methyl- $\alpha$-D-glucoside, but not by other haptenic sugars at similar concentrations $(19,20)$. Similar studies indicated that methyl- $\alpha$-D-galactopyranoside and oligosaccharides containing terminal nonreducing $\alpha$-D-galactopyranosyl units are specific inhibitors of the GS I-B ${ }_{4}$ isolectin $(21,22)$. Although PHA has a complex binding site (23), it is reported that high concentrations of $\mathrm{N}$ -

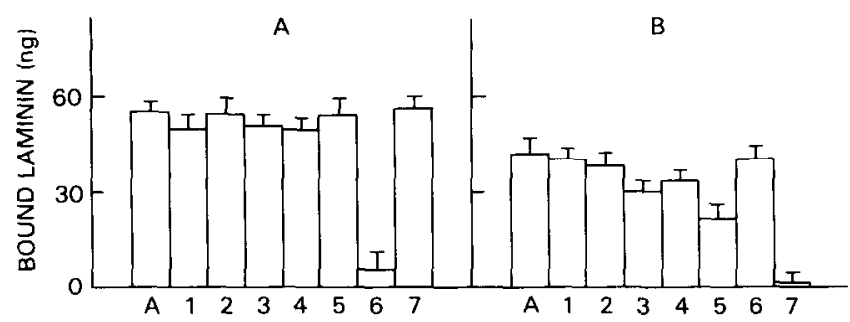

FIG. 4. Binding of ${ }^{125} \mathrm{I}$-laminin to $20 \mu \mathrm{g}$ lectins (A) PHA and (B) WGA in the presence of haptens (1) methyl- $\alpha$-D-galactopyranoside; (2) methyl- $\beta$-D-glucoside; (3) methyl- $\alpha$-D-mannoside; (4) methyl$\beta$-D-galactoside; (5) methyl- $\alpha$-D-glucoside; (6) $N$-acetyl-D-galactosamine; and (7) $N$-acetyl-D-glucosamine. Binding between laminin and PH $\Lambda$ lectin was inhibited by $N$-acetyl-D-galactosamine while the binding of laminin to WGA was inhibited by $N$-acetyl-D-glucosamine. 


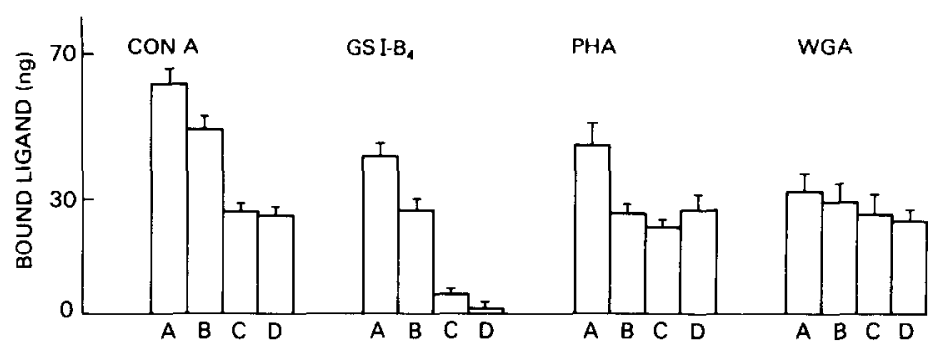

FIG. 5. Comparison of the binding of laminin and fragments to an excess molar ratio of Con $A$, GS I-B 4 , PHA lectins, and wheat germ agglutinin bound to NC. The concentrations of lectins was $1 \mu \mathrm{g} / \mathrm{mm}^{2}$ of filter. (A) Laminin; (B) $\alpha$ fragment; (C) $\mathrm{C}_{1}$ fragment; (D) $\mathrm{P}_{1}$ fragment.

acetyl-D-galactosamine will inhibit its reactivity (24). $N$-Acetyl-D-glucosamine is a specific inhibitor of wheat germ agglutinin. We tested the ability of the NC-filter assay to reproduce the specificity of these lectins for binding to laminin. The data in Figs. 2-4 clearly indicate that the binding of laminin to the immobilized lectins is specific in that Con $\mathrm{A}$ binding was inhibited by a 25 or $50 \mathrm{~mm}$ solution of methyl $\alpha-\mathrm{D}^{-}$ mannopyranoside and methyl- $\alpha$-D-gluco-

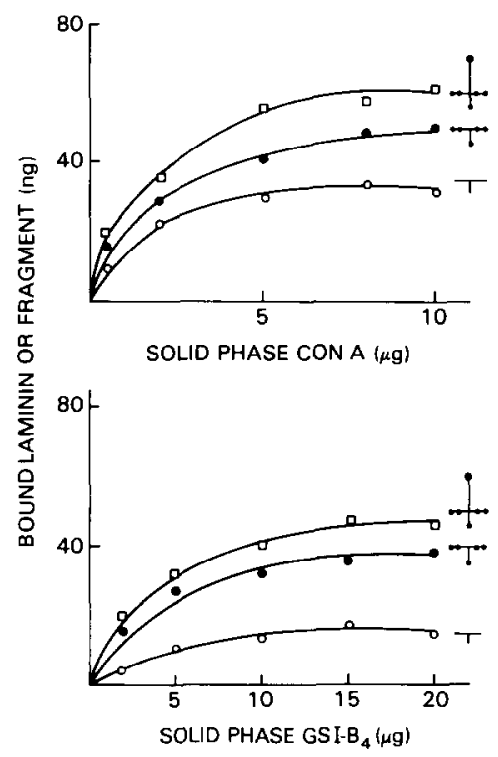

FIG. 6. Binding of laminin and laminin fragments (specific activity $2500 \mathrm{cpm} / \mathrm{ng}$ of protein) to different

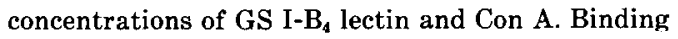
of laminin reached plateau at $10 \mu \mathrm{g}$ of Con $\mathrm{A}$ and 20 $\mu \mathrm{g}$ of GS I-B, lectin on the SCWP nitrocellulose filters. The structure of the fragments is depicted on the right. pyranoside, GS I-B 4 lectin binding was inhibited by methyl- $\alpha$-D-galactopyranoside, PHA lectin binding was inhibited by a 0.5 M solution of $\mathrm{N}$-acetyl-D-galactosamine, and wheat germ lectin binding was blocked by $N$-acetyl-D-glucosamine at the same concentration. The specific sugars involved in the interaction of laminin with different lectins was also confirmed by extracting the filters with $50 \mathrm{~mm}$ solutions of haptenic sugars or $0.5 \mathrm{M}$ solutions of $N$-acetyl-Dglucosamine or $N$-acetyl-D-galactosamine. The respective haptenic sugar, which competitively blocked the lectin binding to laminin, also extracted most of the labeled laminin from the $\mathrm{NC}$, confirming the specificity of the binding reaction.

Whole laminin has the configuration of an asymmetric "cross" with one long arm $(77 \mathrm{~nm})$ and three identical short arms (37 nm) $(7,8)$. The native " $\alpha$ " subunit comprises the three intact short arms of the laminin "cross" (8) and the $\mathrm{C}_{1}$ and $\mathrm{P}_{1}$ fragments consist of the three short arms with the end globular regions reduced or removed $(7,12)$. The $\alpha$ subunit of laminin bound well to five lectins to an extent 15 to $40 \%$ less than to whole laminin. This suggests that the long arm of the laminin "cross" has a considerable amount of carbohydrates. The $\alpha$ subunit and the $\mathrm{C}_{1}$ and the $P_{1}$ fragments differed markedly in their capacity to bind to two of the four lectins. The $\alpha$ subunit, which has the end globular domains, bound to the GS I-B $\mathrm{B}_{4}$ lectin. The $\mathrm{C}_{1}$ and $\mathrm{P}_{1}$ fragments bound to GS I- $\mathrm{B}_{4}$ lectin to a significantly lower extent (60 to $75 \%$ less) compared to the $\alpha$ fragment. We conclude that the end globular domains are 


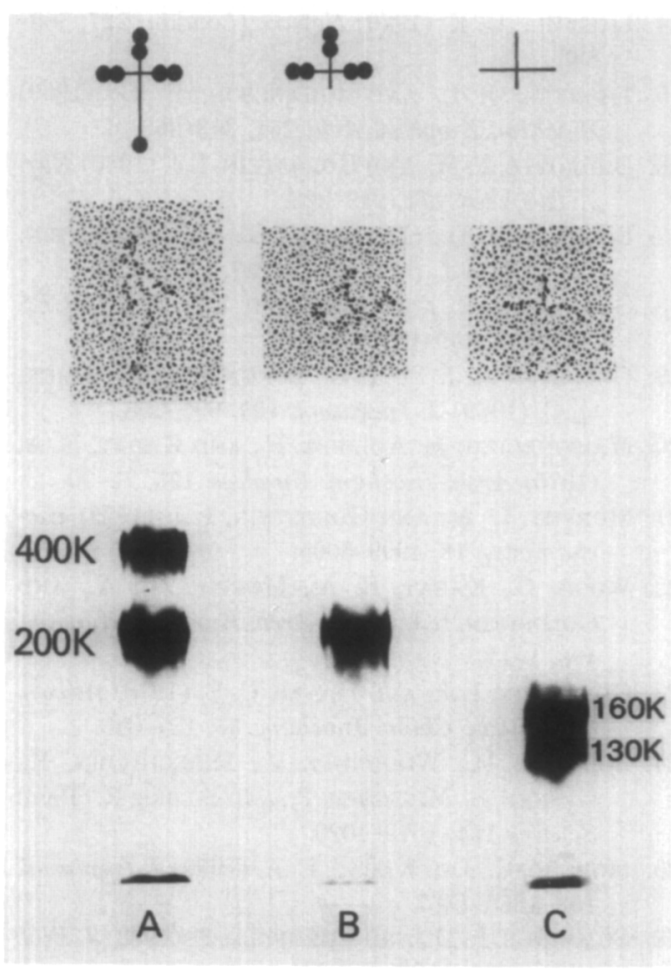

Fig. 7. Sodium dodecyl sulfate (5\%)-gel electrophoresis autoradiography of purified laminin or its fragments. Whole laminin (A), $\alpha$ fragment (B), and $\mathrm{C}_{1}$ fragment (C). A diagrammatic representation of laminin and each of the purified fragments as visualized by rotary-shadowing electron microscopy ( 7 , 8,12 ) is shown on top.

rich in $\alpha$-D-galactopyranosyl end groups. PHA lectin and wheat germ agglutinin bound to the $C_{1}$ or the $P_{1}$ fragment in amounts equivalent to the $\alpha$ subunit. Therefore, we conclude that $N$-acetyl-Dglucosaminyl and galactosaminyl end units are absent or present in low amounts on the globular end regions of the short arms.

On the basis of the lectin-binding studies described in this paper, we may make the following conclusions regarding the glycosyl moieties of laminin.

1. $\alpha$-D-Galactopyranosyl end groups are present as established by the strong re-

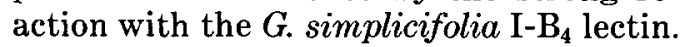
The strong interaction of castor bean ( $R i$ cinus communis) agglutinin, specific for both $\alpha$ - and $\beta$-D-Gal end groups, and the liberation of galactose by coffee bean $\alpha$ galactosidase confirm this conclusion (10).

2. $N$-Acetyl-D-galactosaminyl end groups are absent or present to a very limited extent. This conclusion is based on the poor reactivity of laminin with the lima bean and $D$. biflorus lectins, both of which are quite specific for GalNAc end groups. These lectins also exhibit limited reactivity with $\alpha$-D-galactosyl end groups.

3. $\alpha$-D-Mannopyranosyl residues, most probably substituted at the 2-O-position, are present as evidenced by strong reaction with Con A. Although this lectin also interacts with $\alpha$-D-glucosyl and 2-acetamido2 -deoxy- $\alpha$-D-glucopyranosyl residues, the former sugar is not present in laminin and it would be necessary for the amino sugar to be present as terminal nonreducing units, a rather rare occurrence (25). Inasmuch as the GS II lectin, whose specificity is for GlcNAc end groups, reacted poorly with laminin, it is probable that Con $\mathrm{A}$ is interacting with internal 2-Osubstituted $\alpha$-D-mannopyranosyl residues.

4. The presence of internal $\rightarrow 4)-\beta$-DGlcNAc-(1- units is indicated by the reaction of laminin with wheat germ agglutinin and the $D$. stramonium lectin. The strong reaction with the Datura lectin suggests the presence of $N$-acetyllactosamine $(\beta$-D-Gal- $(1 \rightarrow 4)-D-G l c N A c)$ end units.

5. The strong interaction of laminin with PHA, the Phaseolus vulgaris lectin, indicates the presence of bi-, tri-, or higher antennary oligosaccharide units containing $\alpha$-(NANA)- $\beta$-D-Gal-(1-4)-D-GlcNAc-(1-2)$\alpha$-D-Man- termini $(24,26-29)$.

\section{ACKNOWLEDGMENTS}

This research was supported in part by National Institutes of Health Grant GM29470, and CA20424 from the National Cancer Institute. We thank Dr. D. A. Zopf for helpful discussions. We thank Inger Margulies for performing the electron microscopy.

\section{REFERENCES}

1. Terranova, V. P., Rohrbach, D. H., and Martin, G. R. (1980) Cell 22, 719-726.

2. Sakashita, S., Engvall, E., and Ruoslahti, E. (1980) FEBS Lett. 116, 243-246. 
3. Delrosso, M., Cappelletti, R., Viti, M., VarRnUECHI, S., AND ChIARngI, V. (1981) Biochem. J. 199, 699-704.

4. WoOdley, D. T., RAO, C. N., LiotTa, L. A., Hassel, J. R., Martin, G. R., AND KleinmanN, H. K. (1983) Biochim. Biophys. Acta, in press.

5. Terranova, V. P., RaO, C. N., Kalebic, T., Margulies, I. M. K., And LiotTa, L. A. (1983) Proc. Natl. Acad. Sci. USA 80, 444-448.

6. RaO, C. N., Barsky, S. H., Thrranova, V. P., and LiotTa, L. A. (1983) Biochem. Biophys. Res. Commun. 111, 804-808.

7. Engel, J., Odermatt, E., Engel, A., Madri, J. A., Furthmayr, H., AND TIMPL, R. (1981) $J$. Mol. Biol. 150, 97-120.

8. RaO, C. N., Margulies, I. M. K., Tralka, T. S., Terranova, V. P., Madri, J. A., and Liotta, L. A. (1982) J. Biol. Chem. 257, 9740-9744.

9. HAY, E. D. (1981) in Cell Biology of Extracellular Matrix (Hay, E. D., ed.), pp. 1-39, Plenum, London/New York.

10. Shibata, S., Peters, B. P., Roberts, D. D., GoldSTEIN, I. J., AND LIOTTA, L. A. (1982) FEBS Lett. 142, 194-198.

11. Timpl, R., RoHde, H., Robey, P. G., RenNaRd, S. I., Foldart, J. M., AND Martin, G. R. (1979) J. Biol. Chem. 254, 9933-9977.

12. RaO, C. N., Margulies, I. M. K., Goldfarb, R. H., Madri, J. A., WoOdLeY, D. T., AND LiotTA, L. A. (1982) Arch. Biophys. Biophys. 219, 65-70.

13. Rohde, R. H., Bachinger, H. P., AND Timpl, R. (1980) Hoppe-Seyler's Z. Physiol. Chem. 361, 1651-1660.
14. LAEMMLI, U. K. (1970) Nature (London) 227, 680685.

15. Thorell, J. I, AND Johansson, B. G. (1969) Biochim. Biophys. Acta 251, 363-369.

16. Delmotte, F. M., AND GoldSTEIN, I. J. (1980) Eur. J. Biochem. 112, 219-223.

17. Roberts, D. D., Etzler, M. E., AND Goldstein, I. J. (1982) J. Biol. Chem. 257, 9198-9204.

18. Crowley, J. F., ANd Goldstein, I. J. (1981) FEBS Lett. 130, 149-152.

19. Goldstein, I. J., So, L. L., Yong, Y., ANd CALlier, Q. C. (1969) J. Immunol. 103, 695-698.

20. Maisonrouge-McAuliffe, F., nND KABAT, E. A. (1976) Arch. Biochem. Biophys. 175, 71-90.

21. Murphy, L. A., AND GoldsteIN, I. J. (1979) Biochemistry 18, 4999-5005.

22. Wood, C., Kabat, E. A., Murphy, L. A., AND Goldstein, I. J. (1979) Arch. Biochem. Biophys. 198, 1-11.

23. Goldstein, I. J., ANd Hayes, C. E. (1978) Advan. Carbohydr. Chem. Biochem. 35, 127-340.

24. BorberG, H., WOODRUFF, J., HirSChHORN, R., GeSNer, B., Miescher, P., AND SIl BER, R. (1966) Science 154, 1019-1020.

25. Mokeno, C., ANo Kabat, E. A. (1969) J. Immunol $102,1363-1367$.

26. CUMmings, R. D., AND KoRNFELD, S. (1982) J. Biol. Chem. 257, 11230-11234.

27. KoRnFEld, R., AND KoRnFELd, S. (1970) J. Biol. Chem. 245, 2436-2545.

28. Serafini-Cessi, F., Franceschi, C., and Sperti, S. (1979) Biochem. J. 183, 381-388.

29. Perles, B., Flanagan, M. T., Auger, J., And CRumPtoR, M. J. (1977) Eur. J. Immunol. 7, 613-619. 